\title{
Wiener Lehrsammlungen von Wachspräparaten
}

\author{
Von Erna Lesky
}

\section{E.H.Ackerknecht septuagenario}

Wenn man von Wachspräparaten und von Wien spricht, dann meint man immer die anatomisch-geburtshilflichen Wachspräparate, die Joseph II. in Florenz herstellen und 1786 in seine neu gegründete Medizinisch-chirurgische JosephsAkademie bringen ließ. Diese Sammlung im Josephinum, im heutigen Institut für Geschichte der Medizin, ist so berühmt geworden, daß darüber andere Sammlungen von Wachspräparaten, die im 19. Jahrhundert dazukamen und die z.T. heute noch in Wien existieren, in Vergessenheit gerieten. Ein Ziel dieses Aufsatzes ist es, an diese vergessenen Wachspräparate-Sammlungen zu erinnern und sie kurz vorzustellen. Doch wollen wir zunächst einige Probleme erörtern, die die Präparate im Josephinum gestellt haben und noch weiter stellen.

Dabei kann es sich nicht darum handeln, die Florentiner Entstehungsgeschichte zu wiederholen oder ausführlicher darüber zu sprechen, wie in dem von Peter Leopold 1775 gegründeten Museum für Physik und Naturgeschichte (La Spècola) in Florenz unter der Aufsicht Felice Fontanas (1720-1805) und Paolo Mascagnis (1752-1815) genau nach dem Muster der Florentiner Sammlung auch unsere Wiener Modelle angefertigt wurden. In den letzten eineinhalb Jahrzehnten ist dies mehrmals dargestellt worden ${ }^{1}$. Nun haben diese Wachspräparate in den sechs (bzw. ursprünglich sieben) Sälen des Josephinums seit beinahe 190 Jahren auch eine Wiener Geschichte. Wir möchten gerne von dieser Wiener Geschichte des Florentiner Zwillings erzählen. Die große Schwierigkeit für dieses Unternehmen besteht darin, daß das Archiv der ehemaligen Josephs-Akademie nicht mehr existiert ${ }^{2}$. Wir sind daher auf literarische Quellen angewiesen, als die sich Kataloge der anatomisch-geburtshilflichen Wachspräparate oder Beschreibungen der Josephs-Akademie erweisen.

\section{Die anatomischen Wachspräparate als Instrument der Volksaufklärung}

Wenn das erste monographische Werk über die Wachspräparate, 'Anatomische Tabellen nach der Wachspräparaten-Sammlung der kaiserl. köngl. JosephsAkademie zu Wien. Durchgesehen und beschrieben von Dr. Joseph Scherer ...', erst im Jahre 1817, 31 Jahre nach der Ankunft der Präparate in Wien, zu erscheinen begann, so hängt dies, wie Scherer im Vorwort (S.3) ausdrücklich 
hervorhebt, mit den napoleonischen Kriegen zusammen, die den vollen Einsatz auch und gerade der Professoren der militärärztlichen Akademie erforderten. Joseph Scherer (1750-1844) war von 1806-1823 Professor der Anatomie an der Josephs-Akademie; von 1823-1832 trug er dort Physiologie vor. Eigentlich war das publizistische Unternehmen von dem damals als Zeichner an der Akademie angestellten Wiener Kupferstecher Paul Johann Weindl (1771-1811) ausgegangen. Er hatte bereits seit 1803 begonnen, die Wachspräparate abzuzeichnen, und brachte in dem genannten Werk seine kolorierten Kupfertafeln auf eigene Kosten heraus. Aber bereits 1811 starb Weindl. So kommt es, daß nur mehr der zweite Band (Beginn der Muskellehre) Bilder von Weindls Hand enthält, die drei weiteren, ebenfalls der Muskellehre geltenden Bände aber von Johann Jebmayer illustriert wurden. 1821 blieb das Unternehmen im 5. Band überhaupt stecken. Es hatte sich an das «medicinische Publicum», d.h. an Ärzte, gewendet. Wie wir weiter unten zeigen werden, bezweifelten aber gerade die Ärzte den didaktischen Wert der Wachspräparate für die Anatomie. Daß das Werk Scherers bereits in der Myologie steckenblieb, ist nur ein Zeichen dafür, daß diese Meinung im zweiten Jahrzehnt des 19. Jahrhunderts zum Tragen gekommen war.

So versteht man es, daß sich ein 1837 erschienenes 'Specielles Verzeichnis der anatomisch-physiologischen natürlichen und Wachs-Präparate' des AnatomieProfessors Anton Römer (1786-1842) vornehmlich und der 1857 veröffentlichte Katalog Heinrich Wallmanns ${ }^{3}$ ausschließlich an die «Laien in der Anatomie» wendet und sich nur mehr als «belehrender Wegweiser»versteht wie übrigens auch der 1908 anonym herausgegebene Katalog Franz Bernhard Wildners ${ }^{4}$.

In der Tat haben die Wiener von allem Anfang an der WachspräparatenSammlung im Josephinum ein lebhaftes Interesse entgegengebracht. So konnte bereits die 1795 von Franz II. eingesetzte Untersuchungskommission - wir werden später auf sie zurückkommen - mit deutlicher Indignation feststellen ${ }^{5}$ : «Selbst die mit so vielen Kosten herbeigeschafften Wachs-Praeparate dienen bloß dem Publikum zum Sehen ... » Mag sein, daß deshalb der Einlaß 1802 nur auf jeden zweiten Donnerstag zwischen 10 und 12 Uhr beschränkt war. In dieser Zeit aber waren die Säle der Wachspräparate - damals noch sieben - so überfüllt, «daß man kaum irgendwo Stand fassen kann». ${ }^{6}$ Besonders aber hat sich ein Besucher - es handelt sich um den späteren Berliner Anatomen Karl Asmund Rudolphi (1771-1832) - gewundert ${ }^{7}$ : «Wie groß die Aufklärung in Wien ist, sieht man hier besonders, da eine Menge Frauenzimmer herkommen, um Anatomie zu studieren, und ohne Erröten vor allen Präparaten stehen bleiben.» Fast könnte man meinen, daß der Wunsch in Erfüllung gegangen ist, den der fortschrittliche Arzt Engelbrecht Wichelhausen (1760-1814) 1798 in seinen 
'Ideen über die beste Anwendung der Wachsbildnerei' ${ }^{8}$ so lebhaft zum Ausdruck brachte: « 0 ! wenn doch humane Regenten die Idee auffaßten und zum Unterrichte und Nutzen des Volks auf eine passende, dem Geiste der Zeit gemäße Art die Wachsbildnerei anwendeten, um auch hierdurch manchen aus Unwissenheit entspringenden tragischen Vorfällen vorzubeugen. Was hilft es, daß Kautelen bei unvermeidlichen Übeln unserer Menschennatur in Kompendien stehen ..., wenn das Volk davon keinen Nutzen hat und bei aller sogenannten medicinischen Volksaufklärung ... doch noch der große schätzbare Theil des Volkes, der Landmann und die sogenannten geringern Stände, in den Nebeln der Unwissenheit über die wichtigsten physischen Angelegenheiten stecken.»

Die Liberalität dem «Publicum » gegenüber, von der uns Rudolphi 1802 an der früher zitierten Stelle so Staunenswertes aus dem Josephinum zu berichten weiß, will aber schlecht zu dem reaktionären Geiste passen, der bereits am 24. Dezember 1801 die Privatvorlesungen Franz Josephs Galls (1758-1828) - er gilt heute als Vorläufer der Hirnlokalisationslehre - u. a. deshalb untersagte, weil eben « Frauenzimmer» an ihnen teilnahmen ${ }^{9}$. Auch aus der Einlaß-Ordnung für die Wachspräparatensäle im Josephinum kann man gesellschaftlich relevante Kriterien gewinnen. Noch später, in der Zeit des Neoabsolutismus (1857), wünschte man offensichtlich keine breite Publizität. Zwar waren die Wachspräparate jeden Samstag von 11 bis 13 Uhr (ausgerechnet in der Essenszeit!) zugänglich; die Karten dafür mußte man sich aber zwei Tage (!) vorher, und zwar genau in der Zeit zwischen 11 und 12 Uhr, im Josephinum holen, wobei an eine Person jeweils nur eine Karte ausgegeben wurde. «Individuen des weiblichen Geschlechts» waren gemäß $\S 7^{10}$ überhaupt nicht mehr zugelassen.

Nicht viel besser scheint es mit der Publizität der Wachspräparate auch am Ende des 19. Jahrhunderts gestanden zu haben. Jedenfalls bedauert Johann Habar (1845-1902) ${ }^{11}$ auf das lebhafteste - und er mußte es ja als Mitglied des im Josephinum residierenden Militär-Sanitäts-Komitees wissen -, « daß ein so reichhaltiges Anschauungsmaterial für den Unterricht in der Lehre vom Menschen brach liegt und zur Belehrung weiterer Kreise nicht ausgenützt wird ».

Es bedurfte eines neuen Ansatzes, um die Wiener Wachspräparate als Instrument «medizinischer Volksaufklärung» für weite Kreise des In- und Auslandes neu zu entdecken. Die Kommunikationsmedien und Reproduktionsmöglichkeiten unserer Zeit leisteten dabei wirksame Hilfe. Wichelshausen hätte seine Freude daran, wenn er heute ganze Oberschulklassen und Gruppen von Berufsschulen und viele interessierte Besucher aus nah und fern durch die Säle der Wachspräparate wandern sähe. Aber auch Rudolphi ${ }^{12}$ könnte zufrieden sein. Er hat bereits 1802 die anatomischen Wachspräparate als Lernobjekt für Maler und 
Bildhauer angesprochen. Immer wieder sitzen heute Studenten der Wiener Kunstakademien mit dem Zeichenstift vor ihnen und schulen Auge und Hand an den klassischen Formen anatomischer Wachsplastik des 18. Jahrhunderts. Ihre eigentümliche Schönheit übt auch auf den Heutigen - wir können es oft beobachten - einen seltsamen Zauber aus. Deshalb gilt trotz aller Kritik der Aufklärungsmedizin, was Johann Habart 1896 über sie aussagte: «Diese vielgeschmähte, gelästerte, zum Theil angefeindete, oft absichtlich übersehene Sammlung ist eine der hervorragendsten Sehenswürdigkeiten von Wien.»

\section{Die anatomischen Wachspräparate in der Kritik der Ärzte der Aufklärung}

Es hat geradezu als Zeichen aufklärerischen Rigorosismus zu gelten, daß die Ärzte jener Epoche an der klassischen Schönheit und dem Gestaltungswillen der florentinischen Kästen vorbeisahen, mit denen diese namentlich in den Posen der Großfiguren (Lymphgefäßmann, Muskelmänner) an die Darstellungstradition der großen florentinischen Kunst anknüpften ${ }^{13}$. Geradezu eine Herausforderung an aufklärerischen Sparsinn und an sachliche Nüchternheit bedeutete aber die barocke Pracht und Aufmachung, in denen sich die Wachspräparate darboten. Die weißseidenen Draperien, die blau-atlassenen mit Silberfransen verbrämten Kissen und grünseidenen Vorhänge, die die Wachspräparate umgaben, die Kassetten aus Rosenholz mit goldenen (!) Kanten ${ }^{14}$, all das erregte Unwillen.

Zweifellos spielte dabei auch ein anderes Motiv eine Rolle. Der lombardische Chirurg und seit 1764 Leibchirurg des Erzherzogs Joseph, Alessandro Brambilla (1728-1800 $)^{15}$, auf dessen Initiative der Kaiser 1785 die Josephs-Akademie gegründet hatte, hatte bei ihrer Eröffnung am 7. November eine Rede ${ }^{16}$ gehalten, die die alte Kluft zwischen Ärzten und Chirurgen in voller Breite neuerdings sichtbar machte und die Universitätsmediziner nicht nur in Wien, sondern auch im übrigen Europa provozierte. Brambillas autoritärer Führungsstil sowie die unzulängliche Ausbildung der aus der Akademie hervorgegangenen Militärchirurgen hatte in der ersten Dekade ihres Bestehens nur noch mehr den Unwillen der Ärztekreise erregt. Er entlud sich 1794 in der Schrift des Wiener Spitalarztes und van Swieten-Schülers Franz Xaver Fauken (1740-1794) ${ }^{17}$. Man konnte seine massive Kritik an der Akademie und an Brambilla nicht einfach als Pamphlet abtun. Fauken war durch seinen Entwurf bei der Gründung des Allgemeinen Krankenhauses 1784 sowie durch seine Tätigkeit am St.Marxer Spital eine bekannte und geachtete Persönlichkeit. Der junge Kaiser, Franz II., hat diese, übrigens ihm gewidmete Schrift auch gehörig ernst genommen. Am 19. März 1794 gab er durch ein öffentliches Preisausschreiben das Signal zur 
Reform der Akademie und setzte am 14.Februar 1795 eine eigene Untersuchungskommission dafür ein ${ }^{18}$.

Wir können hier nicht auf die Argumente Faukens gegen die Akademie eingehen. Immerhin will es beachtet sein, daß Fauken die Gründung der Anstalt als Fehlentscheidung der toten Majestät Josephs II. aufgrund schlechter Ratschläge (Brambilla) nach dem Tod Josephs II. hinstellen konnte. Die späteren Kritiker der Akademie haben sich zwar in diesem Punkt von Fauken distanziert; aber sein Urteil über die Wachspräparate, daß sie zum unnützesten Möbel der Erde, zum eigentlichen «Spielwerk für Kinder» $(\mathrm{S} .42,76)^{18}$ a und Laien in der Kunst, gar nicht zum brauchbaren Unterricht für Ärzte und Wundärzte gehörten, stellt in seiner Überspitzung so recht die Gesinnung der Aufklärungsärzte heraus: Hie sparsamste, ein anatomisches Lehrziel anstrebende Nützlichkeit, dort unnütze, barocke Prachtentfaltung, wobei als deren Initiator eben der Günstling Josephs II., Brambilla, angeklagt wurde.

Die Polemik gegen die Josephs-Akademie bzw. gegen die anatomischen Wachspräparate im letzten Jahrfünft des 18. Jahrhunderts ist deshalb so bemerkenswert, weil in ihr sich zwei für die Zeit signifikante Strömungen verflechten: der Kampf der Aufklärung gegen Relikte des Barock und jener zwischen Ärzten und Chirurgen, wobei Brambilla als Galionsfigur für einen ganzen Stand zu gelten hat.

Der vom Kaiser eingesetzten Reformkommission gehörten, wie Belloni bereits erkannt hat, zwei Hauptgruppen an, eine Gruppe von prominenten Ärzten und Universitätsprofessoren wie Johann Peter Frank (1745-1821), der Physiologe Georg Prochaska (1749-1820) und der Botaniker Nicolas Joseph Jacquin (17271817) und eine zweite Gruppe von Professoren der Josephs-Akademie wie der Chirurg Johann Nepomuk Hunczovsky (1752-1798), der Okulist Johann Adam Schmidt (1759-1809) und der Universalmediziner Johann Jakob Plenck (17331807). Das Protokoll dieser Militär-Sanitäts-Kommission, wie der offizielle Titel lautet, hat uns Johann Habart in seinen wesentlichen Teilen erhalten. Das Urteil der Kommission deckt sich in der Substanz, wenn auch durch den behördlichen Stil gemildert, mit dem Faukens und des anonymen Kritikers in der von Johann Jacob Hartenkeil herausgegebenen und im deutschen Sprachraum weit verbreiteten Salzburger Medicinisch-chirurgischen Zeitung ${ }^{19}$. Das Spar-LuxusMotiv beherrscht weithin die Szene, wenn es heißt: «So splendid die Außenseite der akademischen Museen ist, so viele Tausende auch die prächtigen Kabinette, schöne Kästen, die eingelegten Böden, die Wachspräparate mit allen Zeichnungen und seidenen Vorhängen ... dem Staate gekostet haben», so dürfen «die wesentlichen Fehler» doch nicht verhehlt werden, «weil es für den Kenner zu auf- 
fallend ist, wie sehr das Glänzende dem Nützlichen und sogar Nothwendigen allenthalben vorgezogen worden ist. ${ }^{20}$

Was die Kommission des weiteren im einzelnen in ihrem vom 2.Mai 1795 datierten Protokoll ausführt, hat der Anonymus der Salzburger Medicinischchirurgischen Zeitung am 14.Juli $1794^{21}$ «in vierzehn kahlen Worten» ausgesprochen: «Diese prächtige Akademie hat keine anatomische Übungs-Anstalt, weder eine chirurgische, noch medicinische Klinik.»

Luigi Belloni ${ }^{22}$ hat 1974 den Raumplan der Josephs-Akademie aus dem Nachlaß Brambillas publiziert. In der Tat ist auf diesem im Schulgebäude selbst kein Sektionsraum für die Eleven, die künftigen Armeechirurgen, vorhanden. Ein in einiger Entfernung hinter dem Narrenturm gelegenes (im Plan No.25) Sezierkämmerchen konnte nicht ernstlich als «anatomische Übungs-Anstalt» angesprochen werden. Es war «so unbedeutend und klein, daß es für nichts zu rechnen ist».23 Vor diesen historischen Realitäten ist das Urteil Faukens und seiner Nachfolger über die Wachspräparate zu sehen, um seine Härte und Bitterkeit einigermaßen verständlich zu machen.

Die Kampagne gegen Brambilla und die Akademie war längst vorüber, der Prestigestreit zwischen Ärzten und Chirurgen nicht mehr aktuell, als im ersten Drittel des 19. Jahrhunderts die Bildungsreise bei den jungen Ärzten Europas wieder neu in Mode kam. Von diesen ausländischen Ärzten durfte man ein einigermaßen unbefangenes Urteil über die Wachspräparate erwarten. In der Tat gibt es sehr positive Stimmen wie die des englischen Klinikers Richard Bright (1789$1858)^{24}$, «a most splendid collection of anatomical casts in wax», ja sogar überschwengliche wie die des späteren Bonner Geburtshelfers Hermann Friedrich Kilian (1800-1863) ${ }^{25}$, der an «dieser unschätzbaren Sammlung die vollendete Meisterschaft, mit welcher die einzelnen Stücke gefertigt sind und die täuschendste Ähnlichkeit mit der Natur» hervorhebt. Darüber ist aber nicht zu übersehen, daß sich die negative Haltung der Aufklärungsmediziner in modifizierter und differenzierter Form fortsetzt. Hieher gehören die Urteile des schon genannten Karl Asmund Rudolphi aus dem Jahre 1802, des späteren Göttinger Geburtshelfers Johann Friedrich Osiander (1787-1855) ${ }^{26}$ aus dem Jahre 1814 und des Berliner Arztes Wilhelm Horn, der 1828 rundweg feststellt ${ }^{27}$ : «Die Zeit, wo so etwas Interesse erweckte, ist längst vorbei.» Als repräsentativ für diese Linie der Abwertung in der Avantgarde des 19.Jahrhunderts setzen wir das Urteil Karl Asmund Rudolphis ${ }^{28}$ hieher, der als Berliner Anatom der Lehrer des großen Johannes Müller werden sollte: «Manche Figuren sind geradezu idealisch, so erkannte ich den Präparaten über die Nervenstructur gleich die Abbildungen bei Fontana's Buch über das Viperngift ${ }^{29}$; bey den Präparaten von der weiblichen 
Brust war es mir, als ob ich Santorini's Tabulae posthumae ${ }^{30}$ vor mir liegen sähe. Die Gefäße kommen einer schlechten Injection nicht gleich; manche Präparate vom Gehirn leidlich, doch sind mir überall Vicq d'Azyrs ${ }^{31}$ (und gar Soemmerings!) ${ }^{32}$ Abbildungen lieber. Das Auge ist am wenigsten gut in Wachs darzustellen. Die lymphatischen Gefäße scheinen mir zum Theil willkürlich, auch zu groß. Die Muskeln zum Theil recht gut, einige stärker, als ich sie je gesehen habe. Die Knochen recht gut, allein Wachspräparate davon, wenn sie nicht als Kunststücke betrachtet werden sollen, höchst überflüssig, da sie überall und besonders in Wien leicht zu haben sind. Die zur Geburtshülfe gehörenden Präparate sind nicht sehr zu loben, doch werden sie hauptsächlich gesucht. Am besten sind noch die Figuren, welche die Muskeln etc. ganzer Leichname vorstellen. Dem Mahler und Bildhauer kann es hinlänglich seyn, an Wachspräparaten Anatomie zu erlernen, allein für den Arzt und Wundarzt geht es nie an.»

Wir schließen den Chor der jungen und alten Stimmen über die anatomischen Wachspräparate, indem wir die Zelebrität der Aufklärungsmedizin schlechthin hören, Johann Peter Frank, der bereits 1795 bei der Untersuchungskommission mitvotiert hatte und 22 Jahre später, 1817, im 6. Band seines 'System einer vollständigen medicinischen Polizey' ${ }^{33}$ an seiner ablehnenden Stellungnahme festhält. Dabei ist interessant zu erfahren, daß dies Frank nicht aus prinzipiellen didaktischen Gründen tut wie Rudolphi, sondern aus materialtechnischen: «besonders weil die Gebrechlichkeit der Wachspräparate und die in solchen von der Hitze, von der Kälte und, was die Farben betrifft, von dem Tageslicht und vom Schmutze unvermeidlich vorgehenden Veränderungen, Verzerrungen ihrer Dauer, ihrer Genauigkeit und ihrem guten Ansehen sehr enge Grenzen setzen ». Als Beleg dafür führt er die Schäden eben der Wiener Wachspräparaten-Sammlung auf. In der Tat waren gerade während Franks erster Wiener Zeit (1795-1804) 1797, 1801 und später 1805 die ersten Reparaturen an den Wachspräparaten notwendig geworden ${ }^{34}$.

Franks Stellung zu der Wachsplastik seiner Zeit ist aber keineswegs eine undifferenzierte. Während er die anatomische und botanische Wachsbildnerei für unnötig hält, setzt er sich sehr energisch für «das Bossiren gewisser pathologischen Gegenstände in Wachs» ein. Man könne damit das flüchtige Bild einer Krankheit "geschwinde und auf das Genaueste auffangen» und auch Abdrücke davon herstellen.

Wir besitzen heute noch in Wien eine Sammlung pathologischer Wachspräparate, aber derzeit noch nicht im Josephinum. Die wenigen pathologischen Wachspräparate, die es einst dort gab ${ }^{35}$, sind verlorengegangen. Wohl aber besitzt die I. Universitäts-Augenklinik eine Sammlung von Wachspräparaten, die 
Augenkrankheiten darstellen. Sie wird in Kürze in unser Institut überstellt werden. Von ihnen soll im folgenden die Rede sein.

\section{Die Sammlung ophthalmologischer Wachspräparate der I. Universitäts-Augenklinik}

Die Bedeutung der Wiener Schule für die Entwicklung der medizinischen Abbildung ist bisher so gut wie übersehen worden ${ }^{36}$. Wenn diese Darstellung einmal unternommen wird, werden in ihr die Wiener Ophthalmologen einen hervorragenden Platz einnehmen. Vom Schulgründer Georg Joseph Beer (1763-1821) ${ }^{37}$ angefangen über seinen Enkel Eduard Jaeger von Jaxtthal bis zu Maximilian Salzmann (1862-1954) haben viele dieser Okulisten hervorragende zeichnerische Begabung besessen und ihre Lehrbücher vielfach mit kolorierten Zeichnungen von eigener Hand illustriert. Anläßlich des 100-Jahr-Jubiläums der I. Universitäts-Augenklinik haben wir 1962 diese Aquarelle in einer Ausstellung ${ }^{38}$ im Institut für Geschichte der Medizin gezeigt. Darunter befanden sich auch kolorierte Zeichnungen von seltenen Augenkrankheiten, die Georg Joseph Beer bereits seit dem Jahre 1787 angefertigt hatte und die in einem Band mit der Überschrift 'Album der Augenheilkunde' gesammelt sind. Diese kolorierten Zeichnungen dienten dem Unterricht und waren noch im Jahre 1836 im Hörsaal ausgestellt. Der zitierte Band ist als Leihgabe der I. Universitäts-Augenklinik im Institut für Geschichte der Medizin erhalten. Nicht erhalten geblieben sind jedoch die Wachsmodelle vom Auge, die Beer selbst aus didaktischen Gründen angefertigt hatte ${ }^{39}$. Aus all dem sieht man, wie sich bereits an der Beer-Klinik die Tradition herausgebildet hatte, den ophthalmologischen Unterricht mit Hilfe von Zeichnungen und Wachspräparaten seltener Augenkrankheiten so anschaulich wie möglich zu machen.

In dieser Tradition steht auch Beers Schüler Anton Rosas (1791-1855), der 1819-1821 die Augenklinik in Pavia begründet und geleitet hatte und nach dem Tode Beers 1821 die Wiener Klinik übernahm. Bereits 1823 kaufte der Staat auf Initiative Rosas' Wachspräparate von seltenen Augenkrankheiten an, die in ihren schönen, vergoldeten Biedermeier-Glaskästen ebenfalls im Hörsaal aufgestellt wurden. 1836 beschreibt sie der Dissertant von Rosas, Anton Hadwiger ${ }^{40}$, genau. Damals waren es bereits dreißig an der Zahl. Hadwiger nennt auch den Namen des Wachsbossierers. Es war dies der Wiener Wund- und Augenarzt Johann Nepomuk Hoffmayr (auch Hofmayer, Hoffmeyr, Hoffmayer) ${ }^{41}$, der vielleicht noch unter Beer die Kunst des Wachsbossierens gelernt hatte. Im Gegensatz zu den anatomischen Wachspräparaten im Josephinum wurden seine ophthalmologischen Wachsmodelle von den Ärzten der zwanziger Jahre auf das lebhafteste akklamiert. Der deutsche Chirurg Louis Stromeyer (1804-1876) ${ }^{42}$ weiß 
aus dem Jahre 1826 zu berichten, wie sich die damalige Ophthalmologen-Zelebrität Friedrich Jaeger (1784-1872) «sehr über die gelungenen Wachspräparate» freute, und Wilhelm Horn ${ }^{43}$ qualifiziert die Sammlung als «außerordentlich prachtvoll und instruktiv». Er vermerkt aber auch, daß der Augenarzt Hoffmayer seine Kunst «leider wieder teilweise als Geheimnis behandelt» und mit ihr ein gutes Geschäft zu machen versteht: «Ich glaube, eine Darstellung kostet über 70 fl. C.-M.» (= Conventionsmünze).

Die altösterreichische Zentralverwaltung hatte auch ihre guten Seiten. Wenn sich einmal ein didaktisches Hilfsmittel im Unterricht der Metropolitanuniversität als nützlich erwiesen hatte, dann trug sie dafür Sorge, daß mit einem solchen Hilfsmittel auch die anderen unter österreichischer Verwaltung stehenden Universitäten beteilt wurden. Auf diese Weise ist eine Kopie der Wiener Sammlung ophthalmologischer Wachsmodelle an die Augenklinik der Universität Padua gekommen. Dort hat sie Loris Premuda ${ }^{44}$ in einem Winkel entdeckt und einige davon in seinem Bericht 1972 abgebildet. Er schließt seinen Bericht mit der hübschen Wendung: «Florenz war ein Jahrhundert zuvor Wien gegenüber das 'gebende' Element gewesen, nun kam von Wien nach Padua ein künstlerischwissenschaftliches Vermächtnis.»

\section{Mykologische und pomologische Wachskabinette}

Man liest mitunter die Feststellung, daß die Medizinische Polizei Franks im 19. Jahrhundert nicht effizient geworden sei. Wir möchten am konkreten Beispiel den Gegenbeweis für Österreich führen. Im 3. Band seiner Medicinischen Polizey ${ }^{45}$, in dem Frank über die Gefährlichkeit der Pilze handelt, fordert er «eine genauere Bestimmung der Gattungen», um Vergiftungen zu verhüten und eine entsprechende Marktaufsicht zu gewährleisten.

1804 hat der Wiener Arzt Leopold Trattinick (1764-1849) sich daran gemacht, die österreichischen Schwämme zu beschreiben. Er hat dies ganz im Geiste Franks getan. Denn: «Was für ein Opfer könnte auch einem gut gesinnten Staatsbürger zu theuer seyn, wenn es darauf ankommt, Unglücksfälle zu verhüten und dem Vaterlande selbst einen reellen Dienst zu erweisen? ${ }^{46}$ Dieses Buch, das in Lieferungen erschien, enthält genaue Beschreibungen von vierzig Pilzen. Ihm war eine Sammlung von Pilz-Wachsmodellen beigegeben als «mycologisches Cabinet». 1806 war die 1 . Auflage abgeschlossen.

Es ist erstaunlich, mit welcher Raschheit die Österreichische Regierung auf dieses Mittel der Volksaufklärung reagierte, so daß sie bereits 1807 folgendes Regierungsdekret erließ ${ }^{47}$ : «Es ist bei Geistinger Trattinick's vollständiges Werk 
über eßbare Schwämme sammt dazu gehörigen 30 Wachsabbildungen auf Pränumeration zu haben, wodurch den Verwechslungen der guten mit den giftigen Schwämmen sowie jedem Mißbrauche vorgebeugt und dem Gedächtnisse der Marktaufseher nachgeholfen wird.» Sämtliche wundärztlichen Gremien hatten das Werk Trattinicks und die dazugehörigen Pilz-Wachsmodelle anzuschaffen, die von der Firma Reinbold hergestellt wurden. Der Wiener Botaniker Kurt Lohwag ${ }^{48}$ hat solche Wachsmodelle noch 1937 in Zseliz (Slowakei), in Budapest, Triest und selbstverständlich mehrfach in Wien ${ }^{49}$ feststellen können. Ist es bei dieser Verteilerpraxis der österreichischen Behörden verwunderlich, daß auch die Josephs-Akademie 1837 ein «Cabinetto Pomologico in cera» erhielt? Wie alles in dieser Akademie ging auch dieses ins Große. Wie G.Pizzighelli ${ }^{50}$ berichtet, enthielt es 150 Gattungen von Pilzen «d'una bellezza tale, che non si può si di leggieri descrivere». Außerdem enthielt es aber auch noch 300 Gattungen exotische Pflanzen und 200 einheimische und ausländische Früchte.

Wir kennen bereits die Haltung Franks zur botanischen Wachsplastik. Sicher hätte er auch das pomologische Kabinett der Akademie abgelehnt, wenn er es noch erlebt hätte. Desungeachtet bleibt die Tatsache bestehen, daß die österreichische Bürokratie eine der gelehrigsten Schülerinnen Franks war und auf dem mykologischen Sektor seine Medizinische Polizei in einer Weise praktizierte, die er sich kaum erwarten konnte.

\section{Anmerkungen}

${ }^{1}$ Belloni, Luigi, Anatomia plastica. Ciba-Symposium 7 (1959) 229-233; 8 (1960) 84-87, 129-132.

Allmer, Konrad - Jantsch, Marlene, Katalog der josephinischen Sammlung anatomischer und geburtshilflicher Wachspräparate im Institut für Geschichte der Medizin der Universität Wien, Graz/Köln 1965.

Haviland, Thomas N., und Parish, Lawrence Charles, A Brief Account of the Use of Wax Models in the Study of Medicine. Journ. Hist. Med. 25 (1970) 52-76. Lesky, Erna, Anatomia plastica im Wiener Josephinum. Du. Kult. Monatsschr. 31 (1971) 366-377.

Lesky, Erna, Anatomie in Wachs. Zeitmagazin, Nr. 53, 1971, 3-7.

Azzaroli Puccetti, Maria Luisa, La Spècola, the Zoological Museum of the University of Florence. Curator 15 (1972) 93-112.

${ }^{2}$ Erhalten geblieben im Josephinum sind nur: Protocollum sessionum academicarum, 1796-1817. Protokolle der Sitzungen der permanenten Feldsanitätskommission, 1804-1811. Protokoll über die im Examine rigoroso denen Candidaten vorgelegten Skizzen, 1798-1804. 
${ }^{3}$ Verzeichniß der anatomischen und geburtshilflichen Wachspräparate, welche in den Museen der k.k.medizinisch-chirurgischen Josefs -Akademie aufgestellt sind, Wien 1857.

${ }^{4}$ Katalog der Sammlungen anatomischer und geburtshilflicher Wachs-Präparate im k. und k. Josephinum, Wien 1908. Für die Identifizierung vgl. AllmerJantsch (zit. Anm. 1), S. 16.

${ }^{5}$ Habart, Johann, Unser Militär-Sanitätswesen vor hundert Jahren, Wien 1896, S. 75 .

${ }^{6}$ Neuburger, Max, Das alte medizinische Wien in zeitgenössischen Schilderungen, Wien/Leipzig 1921 (zit. im folgenden als Neuburger I), S. 180.

Auch 1828 war nach einem Bericht des Berliner Arztes Wilhelm Horn «alles voll, und die Leute staunten» die Wachspräparate an, «ohne eigentlich zu wissen, daß sie mehr bewundern als die Kunst». Vgl. Neuburger, Max: Die Wiener medizinische Schule im Vormärz, Wien/Berlin 1921 (zitiert im folgenden als Neuburger II), S. 100.

7 Neuburger I, S. 180.

${ }^{8}$ Frankfurt am Main 1798, S. 54f.

${ }^{9}$ Ackerknecht, E.H., und Vallois, H.V., F.J.Gall et sa collection. Mém. du Muséum Nat. d'Hist. Nat., N.S., Sér. A.Zool., Tom.X, Fasc.1, Paris 1955.

Lesky, Erna, Die Wiener medizinische Schule im 19.Jahrhundert, Graz/Köln 1965, S. 22.

10 Wallmann, Heinrich (zit. Anm. 3), S. 2.

11 Habart, Johann (zit. Anm. 5), S. 40.

12 Neuburger I, S.181. Vgl. eine ähnliche Äußerung Johann Friedrich Osianders (1787-1855) aus der Zeit 1814/15 bei Neuburger I, S. 252.

13 Premuda, Loris, Wachsbildnerei und Medizin. Image, Nr.48, 1972, 21, fühlt sich bei Betrachtung der Pose des Lymphgefäßmannes (Katalog Nr.124) an den zum Leben erwachenden Adam von Michelangelo in der Sixtinischen Kapelle erinnert, während ihn die Gestik des Muskelmannes (Katalog Nr. 63) an einen Rhetor der Antike gemahnt.

14 Römer, Anton, S.11. Pizzighelli, Gaetano, Accademia medico-chirurgico-Giuseppina, Wien 1837, S. 49-56.

15 Belloni, Luigi, Lo strumentario chirurgico di Giovanni Alessandro Brambilla. Istituto e Museo di Storia della Scienza Firenze, Mailand 1971. Ders.: Documenti su G.A.Brambilla quale Leibchirurg dell'imperatore Giuseppe II. Clio Medica 7 (1972) 111-115. Ders.: Die Schriften von G. A. Brambilla. In: Lesky, Erna (Hrsg.), Wien und die Weltmedizin, Wien/Köln 1974, S. 32-48.

16 Teilweise abgedruckt bei Neuburger I, 72-81. Die Rede erschien in deutscher, lateinischer, italienischer und französischer Sprache.

17 Fauken, Franz Xaver, Entwurf zu einer Einrichtung in der Heilkunde, Göttingen 1974.

18 Habart, Johann (zit. Anm. 5), S. 40 ff.

${ }^{18 \mathrm{a}}$ Fauken, Franz Xaver (zit. Anm. 17), S. 42 und 76.

${ }^{19}$ Ein Wort zu seiner Zeit, die k.k. medic. chirurg. Josephinische Akademie in Wien betreffend. Med.-chir. Zeitg. 1794/2, 321-334; 1794/3, 49-75. 
${ }^{20}$ Habart, Johann (zit. Anm. 5), S. 74f.

21 1794/3, 61 .

22 Belloni, Luigi (1974, zit. Anm. 15), S. 41.

${ }^{23}$ Habart, Johann (zit. Anm. 5), S. 72.

${ }^{24}$ Neuburger I, S. 223. Bright hielt sich während seiner Kontinentreise 1814/15 in Wien auf.

25 Neuburger II, S. 68. Kilian besuchte Wien im Jahre 1821.

${ }^{26}$ Neuburger I, S. 252.

${ }^{27}$ Neuburger II, S. 100.

28 Neuburger I, S. 181.

${ }^{29}$ Zanobio, Bruno, Le osservazione microscopiche di Felice Fontana sulla struttura dei nervi. Physis 1 (1959) 307-320.

${ }^{30}$ Santorini, Giovanni Domenico, Anatomici summi septemdecim tabulae, quas nunc primum edit atque explicat, iisque alias addit de structura mammarum et de tunica testis vaginali Michael Gerardi, Parma 1775.

${ }^{31}$ Vicq d'Azyr, Félix, Recherches sur la structure du cerveau, du cervelet, de la moelle elongée, de la moelle épinière; et sur l'origine des nerfs de l'homme et des animaux, Paris 1784.

32 Soemmering, Samuel Thomas, De basi encephali et originibus nervorum cranio egredientium libri quinque, Göttingen 1778.

${ }^{33}$ Bd. 6/2, Wien 1817, S.112f.

${ }^{34}$ Allmer-Jantsch (zit. Anm.1), S. 15.

35 Neuburger I, S.181. Rudolphi zählt hier auf «Pocken, Aussatz, allerley Auswüchse, ein Kind mit einer schwarzen warzigen Epidermis u.s.w.»Diese Präparate waren von einem Professor der Akademie ausgeführt worden. Belloni, Luigi (1974, zit. Anm. 15), S. 45.

${ }^{36}$ Einiges habe ich überblicksweise in meinem Buche Die Wiener medizinische Schule im 19. Jahrhundert, Graz/Köln 1965, S. 564f., zusammengefaßt.

${ }^{37}$ Lesky, Erna (zit. Anm. 36), S. 80 f. Koelbing, Huldrych Martin, Augenärztliche Diagnostik der vorophthalmoskopischen Zeit im Bild. Image 60 (1974) $16-23$.

${ }^{38}$ Lesky, Erna, 150 Jahre Wiener Augenheilkunde. Ausstellungskatalog, Wien 1962.

39 Neuburger I, S. 184.

40 Hadwiger, Anton, Geschichte der k.k. Wiener Augenklinik, Wien 1836, S. 21.

41 Im Catalogus chirurgorum des Archivs der Universität Wien finden sich zum Namen folgende Eintragungen: Johannes Hofmajer, geb. in Pottenbrunn, chirurgus Linzii approbatus 21. Aug. 1807. Hofmayer Johannes, geb. 14. April $1783 \mathrm{zu} \mathrm{Linz}$, chirurg. exam. et adprobatus 26. Aug. 1813. Welcher von diesen beiden Hofmayr der Schöpfer der ophthalmologischen Wachspräparate ist, muß mangels anderer Kriterien offen bleiben.

42 Neuburger II, S. $75 \mathrm{f}$.

43 Neuburger II, S. 90.

${ }^{44}$ Premuda, Loris (zit. Anm. 13), S. 22 ff.

45 3. Aufl., Wien 1787, S. 269-293. 
46 Wir benutzten die neue Ausgabe: Die eßbaren Schwämme des Österreichischen Kaiserstaates, Wien 1830, S. VI.

47 Abgedruckt bei Lohwag, Kurt, Das mykologische Wachsfigurenkabinett und die «Pietva fungaja». Österr. Botan.Zschr. 84 (1935) 216.

${ }^{48}$ Lohwag, Kurt, Trattinicks Pilz-Wachsmodelle. Ebda.86 (1937) 131-145.

${ }^{49}$ So in den Sammlungen des Marktamtes der Stadt Wien, in der Porträtsammlung der Österr. Nationalbibliothek sowie in der Tierärztlichen Hochschule.

50 Pizzighelli, Gaetano (zit. Anm. 14), S. 45.

\section{Summary}

At the turn of the 18 th century there were in addition to the well-known collection of Anatomical and Obstetric Wax Models at the Josephinum other collections of wax preparations in Vienna. One of these, the collection of ophthalmologic wax preparations, still exists, while the collection of mycological wax preparations is now lost. After the death of Joseph II. the anatomical wax models were vehemently criticised. The criticism was instigated by physicians of clinical medicine. The strained relations between physicians and surgeons also played an eminent role in this conflict which found its embodiment in the director of the Joseph's Academy, G. A. Brambilla.

Frau Prof. Dr.med. et phil. Erna Lesky

Institut für Geschichte der Medizin

der Universität Wien

Währinger Straße 25

A-1090 Wien 9 